\title{
Perfil do paciente com ceratopatia bolhosa pós-facectomia atendidos em hospital público
}

\author{
Profile of patient with aphakic/pseudopfakic bullous \\ keratopaty attended at public hospital
}

Marcony Rodrigues de Santhiago', Luiz Alberto Molina Monica ${ }^{2}$, Newton Kara-Junior ${ }^{3}$, Beatriz de Abreu Fiúza Gomes ${ }^{4}$, Pedro Moreira Bertino ${ }^{5}$, Maysa Godoy Gomes Mazurek ${ }^{6}$, Yoshifume Yamane ${ }^{7}$, Sergio Henrique Sampaio Meirelles ${ }^{8}$

RESUMO
Objetivo: Identificar e analisar criticamente o perfil dos pacientes com ceratopatia bolhosa
atendidos em um hospital municipal. Métodos: Foi realizada análise retrospectiva de 35
olhos de 35 pacientes em acompanhamento no setor de córnea e doenças externas do
Hospital da Piedade, que apresentavam ceratopatia bolhosa pós-facectomia. Foram ex-
cluídos aqueles onde não se conseguiu determinar características pré e per-operatórias
fundamentais em nossa pesquisa. Esta seleção de pacientes foi realizada durante o perío-
do de janeiro de 2007 a junho de 2007. Resultados: Como principais resultados observou-
se que a ceratopatia bolhosa ocorreu em $22.86 \%$ ( 8 olhos) dos casos pós-facectomia
extracapsular (FEC) e $74.28 \%$ (26 olhos) pós- facoemulsificação. Em $25.71 \%$ ( 9 olhos) foi
documentado rotura de cápsula posterior (RCP) com perda vítrea. Em $94.29 \%$ ( 33 olhos)
a lente intraocular se localizava na câmara posterior, e dois pacientes estavam afácicos. A
acuidade visual de todos os pacientes era pior que $20 / 200$. O tempo médio de evolução
entre a facectomia e o diagnostico de ceratopatia bolhosa foi 11,93 meses (1 - 44 meses).
Conclusão: A análise adequada do endotélio corneano é condição essencial para o sucesso
da cirurgia de catarata com transparência corneal, e que a ceratopatia bolhosa parece
estar mais associada à facoemulsificação. Esta informação deve ser interpretada com
cautela,já que esta relação pode vir do maior numero de facoemulsificações realizadas no
Hospital onde foi realizado o estudo e muitas destas por cirurgiões em treinamento.

Descritores: Doenças da córnea/cirurgia; Transplante de córnea/etiologia; Extração de catarata/efeitos adversos; Complicações pós-operatórias

\footnotetext{
'Estagiário e Pesquisador do Setor de Catarata e do Setor de Cirurgia Refrativa do Hospital da Clínicas da Faculdade de Medicina da Universidade de São Paulo - USP - São Paulo - SP, Brasil;

${ }^{2}$ Chefe do Setor de Córnea e Doenças Externas do Hospital Municipal da Piedade - Rio de Janeiro - RJ, Brasil;

${ }^{3}$ Livre-docente, Professor da Faculdade de Medicina da Universidade de São Paulo - USP - São Paulo (SP), Brasil; Chefe do Setor de Catarata do Hospital das Clinicas Faculdade de Medicina da Universidade de São Paulo - USP - São Paulo - SP, Brasil;

${ }^{4}$ Pós-graduanda (Mestrado) do Hospital Universitário Clementino Fraga Filho da Universidade Federal do Rio de Janeiro - UFRJ - Rio de Janeiro (RJ), Brasil;

${ }^{5}$ Médico Colaborador do Setor Catarata do Hospital Municipal da Piedade - Rio de Janeiro - RJ, Brasil;

${ }^{6}$ Estagiário do Setor de Catarata do Hospital da Clínicas da Faculdade de Medicina da Universidade de São Paulo - USP - São Paulo - SP, Brasil;

${ }^{7}$ Chefe do Setor de Estrabismo do Hospital Municipal da Piedade - Rio de Janeiro - RJ, Brasil;

${ }^{8}$ Chefe do Serviço de Oftalmologia do Hospital Municipal da Piedade - Rio de Janeiro - RJ, Brasil.
}

Trabalho realizado no Hospital Municipal da Piedade - Rio de Janeiro - RJ, Brasil

Recebido para publicação em: 24/12/2008 - Aceito para publicação em 31/8/2009 


\section{INTRODUÇÃO}

$\mathbf{P}$ roduzida por descompensação de função do endotélio corneal, que leva a formação de bolhas epiteliais, a ceratopatia bolhosa é condição clínica crônica e irreversível, sendo uma das principais indicações de transplante de córnea em vários países $^{(1-4)}$. Os estudos vêm mostrando um importante aumento na incidência desta patologia, provavelmente relacionado ao maior número de procedimentos cirúrgicos.

A cirurgia de catarata, que aumentou cerca de $200 \%$ nos últimos 10 anos, contribui de maneira importante dentre as causas de ceratopatia bolhosa em todo o mundo ${ }^{(1)}$. Estudo japonês demonstrou recentemente a facectomia como principal causa de ceratopatia bolhosa no Japão(1), sendo também a principal indicação de transplante penetrante de córnea.

No Brasil há estudos que sugerem este tipo de disfunção corneal como a segunda indicação de transplante atrás apenas do ceratocone ${ }^{(4,5)}$. Um artigo apresentou a ceratopatia bolhosa como $1^{\circ}$ indicação em pacientes entre 71 e $80 \operatorname{anos}^{(6)}$, já outro artigo nacional demonstra esta patologia como principal indicação para aqueles pacientes na fila de transplante de córnea em Sergipe ${ }^{(7)}$. Nos EUA a mesma é responsável por 39\% das indicações de transplante de córnea ficando o ceratocone com $11 \%{ }^{(8)}$.

É por ter impacto social tão importante, que o presente estudo tem como objetivo identificar e analisar criticamente o perfil dos pacientes com ceratopatia bolhosa atendidos no Hospital Municipal da Piedade.

\section{Métodos}

Com aprovação do comitê de ética e pesquisa do Hospital Municipal da Piedade, foi realizado estudo de coorte longitudinal com análise retrospectiva em 35 olhos de 35 pacientes cujo objetivo fora determinar o perfil do paciente com ceratopatia bolhosa pós-cirurgia de catarata, com ênfase nas características per-operatórias. Os pacientes estão em acompanhamento no setor de córnea e doenças externas do Hospital da Piedade.

Foram incluídos no estudo todos os pacientes que apresentaram ceratopatia bolhosa pós-facectomia atendidos no setor de córnea e doenças externas do Hospital da Piedade, entre janeiro de 2007 e julho de 2007. Foram excluídos aqueles onde não se conseguiu determinar características pré e per-operatórias fundamen-
Gráfico 1

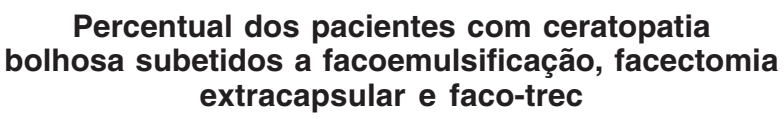

Técnica cirurgica

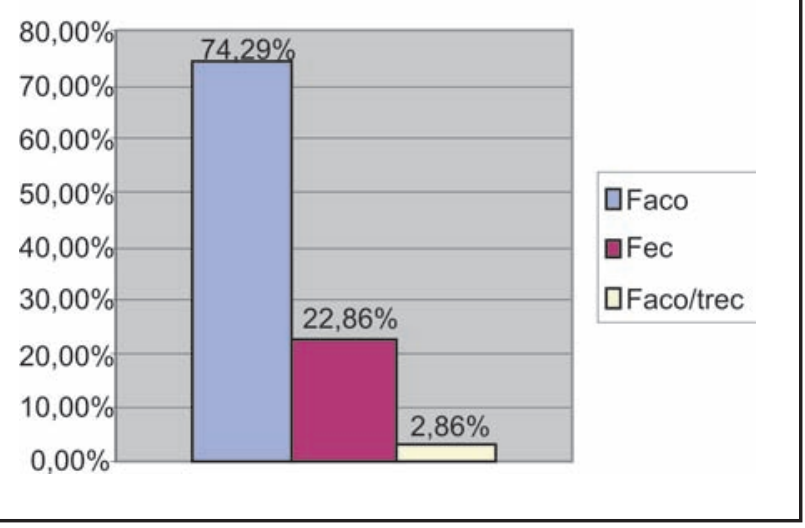

tais em nossa pesquisa. Todos os casos foram diagnosticados por examinador experiente (L.A.M.M.) através de exame em lâmpada de fenda com reflexo especular e microscopia especular no aparelho TOPCOM SP2000P.

Determinou-se perfil do paciente através de análise investigativa de prontuário. Foi identificado quando e onde foi realizada a cirurgia de catarata, a técnica cirúrgica empregada, se realizada por cirurgião residente ou não, material viscoelástico utilizado, se pseudofácico ou afácico, localização da lente intraocular (LIO) e se havia desvio da LIO. Além do tempo de evolução entre a cirurgia e o diagnóstico de ceratopatia bolhosa.

A partir da análise dos dados obtidos, traçamos o perfil do paciente com determinada patologia avaliado no setor de córnea e doenças externas do Hospital da Piedade.

\section{Resultados}

Os dados referentes aos 35 olhos dos 35 pacientes avaliados estão agrupados no Quadro 1.

$\mathrm{Na}$ presente análise observou-se que a ceratopatia bolhosa ocorreu em $22.86 \%$ ( 8 olhos) dos casos pós-facectomia extracapsular (FEC) e 74.28\% (26 olhos) pós-facoemulsificação. Em um dos casos, foi realizado trabeculectomia associada à facoemulsificação (Gráfico 1). 
Quadro 1

Principais características analisadas nos 35 olhos incluídos no estudo

\begin{tabular}{|c|c|c|c|c|c|c|c|c|c|c|c|c|}
\hline Paciente & $\begin{array}{l}\text { Técnica } \\
\text { cirúrgica }\end{array}$ & $\begin{array}{r}\text { Posição } \\
\text { da LIO }\end{array}$ & RCP & Sexo & $\begin{array}{c}\text { Idade } \\
\text { visual } \\
\text { LogMAR }\end{array}$ & Acuidade & $\begin{array}{c}\text { Data } \\
\text { da cirurgia }\end{array}$ & Diagnóstico & $\begin{array}{l}\text { Tempo } \\
\text { meses }\end{array}$ & DM & Guttata" & PEX \\
\hline 1 & fec & C.P. & S & $\mathrm{F}$ & 57 & $>1.0$ & out/01 & mai/05 & 43 & $\mathrm{~N}$ & $\mathrm{~S}$ & $\mathrm{~N}$ \\
\hline 2 & faco & C.P. & $\mathrm{N}$ & $\mathrm{F}$ & 70 & $>1.0$ & jul/00 & $\mathrm{dez} / 00$ & 5 & $\mathrm{~N}$ & $\mathrm{~N}$ & $\mathrm{~N}$ \\
\hline 3 & fec & C.P. & $\mathrm{S}$ & $\mathrm{F}$ & 61 & $>1.0$ & $\mathrm{fev} / 02$ & $\mathrm{jan} / 03$ & 11 & $\mathrm{~S}$ & $\mathrm{~S}$ & $\mathrm{~N}$ \\
\hline 4 & faco/trec & C.P. & $\mathrm{N}$ & $\mathrm{F}$ & 69 & $>1.0$ & out/04 & ago/05 & 10 & $\mathrm{~N}$ & $\mathrm{~N}$ & $\mathrm{~S}$ \\
\hline 5 & faco & C.P. & $\mathrm{N}$ & $\mathrm{F}$ & 67 & $>1.0$ & $\mathrm{ago} / 03$ & set/03 & 1 & $\mathrm{~N}$ & $\mathrm{~S}$ & $\mathrm{~N}$ \\
\hline 6 & faco & C.P. & $\mathrm{N}$ & $\mathrm{F}$ & 72 & $>1.0$ & out $/ 05$ & jul/06 & 9 & $\mathrm{~N}$ & $\mathrm{~N}$ & $\mathrm{~N}$ \\
\hline 7 & fec & C.P. & $\mathrm{S}$ & $\mathrm{F}$ & 80 & $>1.0$ & $\mathrm{mar} / 05$ & $\mathrm{mar} / 06$ & 12 & $\mathrm{~N}$ & $\mathrm{~S}$ & $\mathrm{~N}$ \\
\hline 8 & faco & C.P. & $\mathrm{N}$ & $\mathrm{F}$ & 65 & $>1.0$ & ago/04 & $\mathrm{jan} / 06$ & 17 & $\mathrm{~N}$ & $\mathrm{~N}$ & $\mathrm{~N}$ \\
\hline 9 & faco & C.P. & $\mathrm{N}$ & $\mathrm{F}$ & 67 & $>1.0$ & out/04 & set/05 & 11 & $\mathrm{~N}$ & $\mathrm{~N}$ & $\mathrm{~N}$ \\
\hline 10 & faco & C.P. & $\mathrm{N}$ & $\mathrm{F}$ & 71 & $>1.0$ & $\mathrm{mar} / 04$ & $\mathrm{abr} / 05$ & 13 & $\mathrm{~N}$ & $\mathrm{~N}$ & $\mathrm{~N}$ \\
\hline 11 & fec & C.P. & $\mathrm{S}$ & $\mathrm{F}$ & 69 & $>1.0$ & $\mathrm{jan} / 06$ & ago/06 & 7 & $\mathrm{~S}$ & $\mathrm{~N}$ & $\mathrm{~N}$ \\
\hline 12 & faco & C.P. & $\mathrm{N}$ & $\mathrm{F}$ & 72 & $>1.0$ & $\mathrm{jul} / 01$ & $\mathrm{dez} / 01$ & 5 & $\mathrm{~N}$ & $\mathrm{~N}$ & $\mathrm{~N}$ \\
\hline 13 & faco & C.P. & $\mathrm{N}$ & $\mathrm{F}$ & 73 & $>1.0$ & $\mathrm{jul} / 03$ & mai/04 & 11 & $\mathrm{~S}$ & $\mathrm{~N}$ & $\mathrm{~N}$ \\
\hline 14 & $\mathrm{fec}$ & C.P. & $\mathrm{N}$ & $\mathrm{F}$ & 81 & $>1.0$ & $\mathrm{abr} / 05$ & mai/06 & 13 & $\mathrm{~N}$ & $\mathrm{~S}$ & $\mathrm{~N}$ \\
\hline 15 & faco & C.P. & $\mathrm{N}$ & $\mathrm{F}$ & 69 & $>1.0$ & set/03 & out/04 & 13 & $\mathrm{~N}$ & $\mathrm{~N}$ & $\mathrm{~N}$ \\
\hline 16 & faco & C.P. & $\mathrm{N}$ & $\mathrm{F}$ & 61 & $>1.0$ & $\mathrm{jan} / 06$ & $\mathrm{abr} / 06$ & 3 & $\mathrm{~N}$ & $\mathrm{~N}$ & $\mathrm{~N}$ \\
\hline 17 & fec & C.P. & $\mathrm{N}$ & $\mathrm{M}$ & 70 & $>1.0$ & $\mathrm{mar} / 01$ & set/02 & 18 & $\mathrm{~N}$ & $\mathrm{~S}$ & $\mathrm{~N}$ \\
\hline 18 & faco & C.P. & $\mathrm{N}$ & $\mathrm{F}$ & 73 & $>1.0$ & $\mathrm{fev} / 07$ & $\mathrm{abr} / 07$ & 2 & $\mathrm{~N}$ & $\mathrm{~N}$ & $\mathrm{~N}$ \\
\hline 19 & faco & afácico & $\mathrm{S}$ & $\mathrm{F}$ & 65 & $>1.0$ & $\mathrm{mar} / 05$ & out/05 & 7 & $\mathrm{~N}$ & $\mathrm{~N}$ & $\mathrm{~N}$ \\
\hline 20 & faco & C.P. & $\mathrm{N}$ & $\mathrm{F}$ & 54 & $>1.0$ & $\mathrm{jan} / 06$ & $\mathrm{fev} / 07$ & 13 & $\mathrm{~N}$ & $\mathrm{~N}$ & $\mathrm{~N}$ \\
\hline 21 & faco & C.P. & $\mathrm{N}$ & $\mathrm{F}$ & 61 & $>1.0$ & $\mathrm{mar} / 03$ & $\mathrm{dez} / 03$ & 9 & $\mathrm{~S}$ & $\mathrm{~N}$ & $\mathrm{~N}$ \\
\hline 22 & faco & C.P. & $\mathrm{N}$ & $\mathrm{F}$ & 79 & $>1.0$ & $\mathrm{fev} / 05$ & $\mathrm{fev} / 06$ & 12 & $\mathrm{~N}$ & $\mathrm{~N}$ & $\mathrm{~N}$ \\
\hline 23 & faco & C.P. & $\mathrm{N}$ & $\mathrm{M}$ & 80 & $>1.0$ & $\mathrm{abr} / 04$ & $\mathrm{mar} / 06$ & 23 & $\mathrm{~N}$ & $\mathrm{~N}$ & $\mathrm{~N}$ \\
\hline 24 & fec & C.P. & $\mathrm{N}$ & $\mathrm{F}$ & 65 & $>1.0$ & nov/05 & jul/06 & 8 & $\mathrm{~N}$ & $\mathrm{~S}$ & $\mathrm{~N}$ \\
\hline 25 & faco & C.P. & $\mathrm{N}$ & $\mathrm{F}$ & 77 & $>1.0$ & $\mathrm{mai} / 06$ & $\mathrm{mar} / 07$ & 10 & $\mathrm{~N}$ & $\mathrm{~N}$ & $\mathrm{~N}$ \\
\hline 26 & faco & afácico & $\mathrm{S}$ & $\mathrm{F}$ & 72 & $>1.0$ & nov/04 & jun/05 & 5 & $\mathrm{~N}$ & $\mathrm{~N}$ & $\mathrm{~N}$ \\
\hline 27 & faco & C.P. & $\mathrm{N}$ & $\mathrm{F}$ & 81 & $>1.0$ & $\mathrm{abr} / 05$ & ago/05 & 4 & $\mathrm{~S}$ & $\mathrm{~N}$ & $\mathrm{~N}$ \\
\hline 28 & faco & C.P. & $\mathrm{N}$ & $\mathrm{F}$ & 79 & $>1.0$ & $\mathrm{ago} / 05$ & set/05 & 1 & $\mathrm{~N}$ & $\mathrm{~S}$ & $\mathrm{~N}$ \\
\hline 29 & faco & C.P. & $\mathrm{S}$ & $\mathrm{F}$ & 64 & $>1.0$ & set/03 & $\mathrm{dez} / 04$ & 15 & $\mathrm{~N}$ & $\mathrm{~N}$ & $\mathrm{~N}$ \\
\hline 30 & faco & C.P. & $\mathrm{S}$ & $\mathrm{M}$ & 78 & $>1.0$ & $\mathrm{mar} / 04$ & $\mathrm{jan} / 05$ & 10 & $\mathrm{~S}$ & $\mathrm{~N}$ & $\mathrm{~N}$ \\
\hline 31 & faco & C.P. & $\mathrm{N}$ & $\mathrm{F}$ & 54 & $>1.0$ & $\mathrm{mar} / 05$ & $\mathrm{ago} / 05$ & 5 & $\mathrm{~N}$ & $\mathrm{~N}$ & $\mathrm{~N}$ \\
\hline 32 & fec & C.P. & $\mathrm{N}$ & $\mathrm{F}$ & 71 & $>1.0$ & out/04 & out/05 & 12 & $\mathrm{~N}$ & $\mathrm{~S}$ & $\mathrm{~N}$ \\
\hline 33 & faco & C.P. & $\mathrm{N}$ & $\mathrm{F}$ & 63 & $>1.0$ & $\mathrm{jan} / 03$ & $\mathrm{mai} / 05$ & 28 & $\mathrm{~N}$ & $\mathrm{~N}$ & $\mathrm{~N}$ \\
\hline 34 & faco & C.P. & $\mathrm{S}$ & $\mathrm{F}$ & 75 & $>1.0$ & $\mathrm{jul} / 02$ & $\mathrm{abr} / 03$ & 9 & $\mathrm{~N}$ & $\mathrm{~N}$ & $\mathrm{~N}$ \\
\hline 35 & faco & C.P. & $\mathrm{N}$ & $\mathrm{F}$ & 79 & $>1.0$ & mai/05 & nov/05 & 6 & $\mathrm{~N}$ & $\mathrm{~N}$ & $\mathrm{~N}$ \\
\hline
\end{tabular}

faco $=$ facoemulsificação; fec $=$ facectomia extracapsular; C.P. = cápsula posterior; C.A. = cápsula anterior; $\mathrm{S}=$ sim; $\mathrm{N}=$ não; $\mathrm{F}=$ feminino; $\mathrm{M}=$ masculino; $\mathrm{DM}=$ diabetes mellitus; $\mathrm{PEX}$ = síndrome de pseudoesfoliação

Em 25.71\% (9 olhos) foi documentado rotura de cápsula posterior (RCP) com perda vítrea. Em 94.29\% (33 olhos) a LIO se localizava na câmara posterior, e dois pacientes estavam afácicos. Em nenhum de nossos casos foi implantado LIO de câmara anterior.

A média de idade dos pacientes de nosso estudo foi de 69 anos (51 - 81 anos), havendo importante predominância desta determinada condição clínica entre as mulheres nos casos documentados no setor de córnea e doenças externas do Hospital da Piedade.

Em média, encontramos um tempo de 11,93 meses $(1-44$ meses $)$ de evolução desde a cirurgia até o diagnóstico de ceratopatia bolhosa.

Todos os pacientes apresentavam acuidade visual na tabela $\log$ MAR > 1.0 (Acuidade visual <20/200) nos olhos estudados.

Em todos os olhos foram implantadas LIOs rígidas de PMMA (3 peças) no saco capsular, a não ser nos casos onde houve rotura de cápsula posterior, quando a LIO foi colocada no sulco ciliar .

Como viscoelástico, a Metilcelulose ${ }^{\circledR}$, de característica dispersiva, foi utilizada em todas as facectomias.

Em 29 olhos as cirurgias foram realizadas por cirurgiões em treinamento (residentes). 
Como características pré-operatórias, identificamos diabetes mellitus (6 olhos), síndrome de pseudoesfoliação (1 olho ) e córnea "gutatta" (9 olhos).

\section{Discussão}

Atualmente sabe-se que a perda endotelial póscirurgia de catarata é 2,5 a 8 vezes maior que a perda anual, devido ao envelhecimento de um olho não operado, independentemente da técnica cirúrgica utilizada. Foi baseado na importância do endotélio corneano ${ }^{(9)}$, que ocorreram muitas descobertas e melhoramentos tecnológicos em facetomia. Incisões menores, técnicas de facoemulsificação com menor utilização de energia ultrassônica, além de novos materiais viscoelásticos são alguns dos exemplos de esforço nesse sentido ${ }^{(10-13)}$.

Revisando detalhadamente os arquivos dos pacientes do presente estudo, observamos a presença de fatores de risco pré-operatórios que devem ser, de maneira consistente, considerados na hora de se indicar a facectomia, principalmente no que concerne ao fato de ser um hospital com cirurgiões em treinamento. A maioria dos olhos que desenvolveram ceratopatia bolhosa foi submetida à cirurgia com cirurgião residente ( 29 olhos).

Diabetes mellitus (6 olhos), síndrome de pseudoesfoliação (1 olho), cirurgia prévia do segmento anterior e córnea "gutatta" ( 9 olhos) são definitivamente fatores que tornam o endotélio mais susceptível ao dano cirúrgico ${ }^{(9)}$.

Mesmo em uma amostra reduzida desta análise, foi possível notar uma maior prevalência de diabetes mellitus em pacientes com ceratopatia bolhosa, em relação a população brasileira ${ }^{(14)}$.

Além disso, é importante ressaltarmos que $1 / 4$ de nossos casos ocorreram em pacientes que apresentavam córnea "gutatta". Dos 9 olhos com tal condição prévia , 7 foram submetidos a facectomia extracapsular e apenas 2 à facoemulsificação. É por constatações desse tipo, que não devemos nos abster de uma indicação de cirurgia tríplice em paciente que apresente catarata associada à gutatta avançada ${ }^{(15)}$.

As LIOs de PMMA, usadas em todos os pacientes pseudofácicos do estudo, podem gerar reações inflamatórias devido à ativação do complemento, o que vai determinar a biocompatibilidade do material. Pela natureza hidrofóbica do PMMA, pode ocorrer dano tissular significativo em caso de toque da LIO no endotélio da córnea ${ }^{(16)}$.

A metilcelulose ${ }^{\circledR}$ parece não ser tão eficaz em proteger o endotélio quanto os outros viscoelásticos dispersivos $^{(10)}$.

Assim como recente pesquisa com mais de 900 pacientes realizado no Japão ${ }^{(1)}$, observamos que a ceratopatia bolhosa ocorreu mais comumente pósfacoemulsificação em $74.28 \%$ (26 olhos $)^{(1,9-10)}$. A facoemulsificação é atualmente realizada por mais de $90 \%$ dos cirurgiões de catarata brasileiros ${ }^{(17)}$.

Sabemos que é fator fundamental na formação de edema pós-operatório o número de partículas ricocheteando na câmara anterior ${ }^{(9-10)}$. Atualmente, se sabe ainda, que incisões menores tendem a provocar menor perda endotelial $^{(11),}$ assim como incisões esclerais ${ }^{(12)}$. Outro parâmetro importante é o tempo de ultrassom (US) e energia liberada na câmara anterior que está associado ao desenvolvimento de edema corneano, o que tem feito com que as técnicas de chopping ganhem cada vez mais espaço, na tentativa de se minimizar o trauma cirúrgico ao endotélio ${ }^{(13,18)}$. O presente estudo, no entanto,não foi capaz de detalhar tais condições em todos os pacientes, sendo assim, dados como tempo de US e tipo de incisão, não foram incluídos nesta análise de perfil de nossos pacientes.

A ruptura de cápsula posterior é a complicação peroperatória mais frequente em cirurgia de catarata, ocorrendo em cerca de $2 \%$ dos $\operatorname{casos}^{(19)}$. Em nosso estudo, esta complicação ocorreu em $25.71 \%$ dos casos o que sugere um risco relativamente maior de ocorrer ceratopatia bolhosa quando exposto a este determinado fator.

Em média, encontramos um tempo de 11.87 meses $(1-44$ meses $)$ de evolução desde a cirurgia até o diagnóstico de ceratopatia bolhosa. A média de idade dos pacientes de nosso estudo foi de 69 anos (51 - 81 anos), havendo importante predominância de mulheres nos casos documentados no setor de córnea e doenças externas do Hospital da Piedade. Outros estudos já demonstraram uma maior prevalência em faixa etária acima de 65 anos, mas diferem de nossa análise, mostrando uma prevalência maior em mulheres apenas discreta ${ }^{(1,10)}$.

Notou-se ainda que, em pacientes com idade igual ou superior a setenta anos, a evolução para ceratopatia bolhosa foi mais rápido. Média de 9.3 meses comparada à média de 12.5 meses dos pacientes com menos de 70 anos.

Com a proposta de analisar criticamente e determinar o perfil dos pacientes com ceratopatia bolhosa atendidos no Hospital da Piedade, nosso estudo não tem o desenho e alcance suficientes para determinar fatores de risco para patologia tão importante e com significativo impacto social na vida dos pacientes.

Conclui-se, no entanto, que a análise adequada do endotélio corneano é condição "sine qua non" para o sucesso da cirurgia de catarata com transparência 
corneal, e que a ceratopatia bolhosa parece estar mais associada à facoemulsificação, o que deve ser interpretado com cautela, já que esta relação pode vir do maior número de facoemulsificações realizadas no Hospital onde foi realizado o estudo e muitas ainda realizadas por cirurgiões inexperientes.

Com a certeza de que mais estudos nesse sentido devam ser realizados, estimulamos os hospitais com cirurgiões em treinamento a pesquisar sobre suas complicações, definindo claramente as principais características destas, bem como de seus pacientes, com a finalidade de diminuir a presença destes nas filas de transplante de córnea.

\section{Abstract}

Objective: Analyze and identify critically the profile of patients with aphakic/pseudophakic bullous keratopathy attended at a public hospital. Methods: Retrospective analysis of 35 eyes of 35 patients with diagnosis of bullous keratopathy, which has been followed in the department of cornea and external diseases of Hospital da Piedade. The one whose pre and per operative data we did not find were excluded. This selection of patients was done from January to June of 2007. Results: As main results we found that bullous keratopathy occurred in $22.86 \%$ (8 eyes) of cases after extracapsular phakectomy and $74.28 \%$ (26 eyes) after phacoemulsification. Rupture of posterior capsule and vitreous loss was documented in $25.71 \%$ (9 eyes). In $94.29 \%$ (33eyes) the intraocular lenses was in posterior chamber, and two patients were aphakic. The visual acuity of all patients was worse than 20/200. The mean time from phakectomy to diagnosis of bullous keratopathy was 11.93 months (1-44 months). Conclusion: Adequate analysis of corneal endothelium is an essential condition to obtain success of cataract surgery with corneal transparency, and bullous keratopathy seems to be more related to phacoemulsification. That should be read with caution since this relation may occur because of the higher number of phacoemulsifications that are practiced in that Hospital where the study was conducted and many of them was performed by training surgeons.

Keywords: Corneal diseases/surgery; Corneal transplantation/etiology; Cataract extraction/adverse effects; Postoperative complications.

\section{REFERÊNCIAS}

1. Shimazaki J, Amano S, Uno T, Maeda N, Yokoi N . National survey on bullous keratopathy in Japan. Cornea. 2007;26(3):274-8.
2. Al-Towerki AE, Gonnah el-S, Al-Rajhi A, Wagoner MD. Changing indications for corneal transplantation at the King Khaled eye specialist hospital (1983- 2002). Cornea. 2004;23(6):584-8.

3. Al-Yousuf N, Mavrikakis I, Mavrikakis E, Daya SM. Penetrating keratoplasty: Indications over a 10 year period. $\mathrm{Br}$ J Ophthalmol. 2004;88(8):998-1001.

4. Calix Netto MJ, Giustina ED, Ramos GZ, Peccini RF, Sobrinho M, de Souza LB. Major indications for corneal penetrating keratoplasty at a reference service in Sao Paulo state (Sorocaba - SP, Brazil). Arq Bras Oftalmol. 2006;69(5):661-4.

5. Fabris C, Correa ZM, Marcon A, Castro TN, Marcon IM, Pawlowski C . Estudo retrospectivo dos transplantes penetrantes de córnea da Santa Casa de Porto Alegre. Arq Bras Oftalmol. 2001; 64(5):449-53.

6. Flores VG, Dias HL, Castro, RS. Indicações para ceratoplastia penetrante no Hospital das Clínicas-UNICAMP. Arq Bras Oftalmol. 2007;70(3):505- 8

7. Araújo AA, Melo GB, Silva RL, Araújo Neta VM . Perfil epidemiológico dos pacientes na lista de espera para transplante de córnea no Estado de Sergipe. Arq Bras Oftalmol. 2004, 67(4):613- 6

8. Dobbins KR, Price FW Jr, Whitson WE. Trends in the indications for penetrating keratoplasty in the midwestern United States. Cornea. 2000; 19(6):813-6.

9. Kwitko S. Endotélio e cirurgia da catarata: grandes desafios. Arq Bras Oftalmol. 2000; 63(3):235-9 .

10. Aquavella JV, Willians ZR, McCormick GJ, Singer DE. Pseudophakic bullous keratophathy. E-Medicine [ Internet]. [Updated 2005 May]. Available from. http:// www.emedicine.com/oph/topic107.htm

11. Dick HB, Kohnen T, Jacobi FK. Long-term endothelial cell loss following phacoemulsification through a temporal incision. J Cataract Refract Surg 1996; 22(1):63-71.

12. Crema AS; Walsh A; Yamane R. Perda de células endotelias após facoemulsificação por incisões auto selantes escleral superior e corneana temporal. Rev Bras Oftalmol. 1997; 56(1):17-26

13. Chang D. Phaco Chop. Thorofare (NJ): Slack; 2004.

14. Malerbi DA, Franco LJ. Multicenter study of the prevalence of diabetes mellitus and impaired glucose tolerance in the urban Brazilian population aged 30-69 yr. Diabetes Care. 1992; 15(11): 1509-16.

15. Molina LA . Córnea guttata como fator de predisposição para ceratopatia bolhosa. Arq Bras Oftalmol; 1998; 61(4): 430.

16. Leaming DV. Practice styles and preferences of ASCRS members - 2003 survey. J Cataract Refract Surg. 2004; 30(4):892-900.

17. Santhiago MR, Gomes BF, Gafree FP, Varandas VS, Costa Filho AA. Tendências evolutivas dos cirurgiões de catarata presentes no IV Congresso Brasileiro de Catarata e Cirurgia Refrativa. Rev Bras Oftalmol. 2009; 68(1):13-7.

18. Centurion V. Complicações per-operatórias durante a facoemulsificação. Rev Bras Oftalmol. 1999; 58(9): 687- 91.

19. Rezende F. Cirurgia de catarata. Rio de Janeiro: Cultura Médica; 2002

\section{Endereço para correspondência: \\ Rua Teodoro Sampaio, ${ }^{\circ} 498$ apto 35 - Pinheiros \\ CEP 05406-000 - São Paulo - SP}

E mail: marconysanthiago@hotmail.com 